frequency in the past four weeks respectively, at baseline. Calculating change scores over time, we assessed whether changes in social media belonging, were associated with changes in drinking status, and whether changes in frequency of use of social media sites were related to changes in drinking frequency, using multinomial logistic and linear regression model. Analyses were repeated with number of times binge drinking $(5+$ drinks $)$ in the past four weeks, on a sub-sample of participants aged 16-19. Models adjusted for sex, age, household tenure, urban/rural area, educational/work status, baseline social media use/frequency and number of friends.

Results At baseline, those who belonged to a social media site were over four times more likely $(\mathrm{OR}=4.6295 \% \mathrm{CI}$ (3.61-5.91)) to be a drinker versus non-drinker. Those who chatted for longer on social media sites were more likely to have drank at least once a week compared with not drinking in the past month (none vs. 1-3 hours (2.31(1.45$3.68)$, 4-6 hours 2.60(1.44-4.72), 7 hours or more 5.92 (2.86-12.28). Among participants aged 16-19, a similar gradient was found with higher odds of binge drinking three or more times in the past month, with greater chat frequency (e.g. no hours chatting vs. 7 hours or more 6.29 (2.50-15.82)). Over time, becoming new social media users increased the odds of becoming a new drinker (1.77 (1.043.03)) versus no change in drinking status. Participants who became more frequent users of social media sites, were more likely to drink more frequently $(\beta=0.13,95 \%$ CI $(0.09-0.17))$, and binge drink $(\beta=0.21$, 95\% CI $(0.12-$ $0.31)$ ) more frequently across waves.

Conclusion Increasing social media use was associated with greater alcohol consumption; mechanisms behind this relationship should be investigated. Alcohol-related content on the internet should be monitored.

\section{P89 TACKLING LOCAL ALCOHOL-RELATED HARMS IN THE NIGHT TIME ECONOMY: A PROCESS EVALUATION WITH A COMPLEX SYSTEMS PERSPECTIVE}

${ }^{1} \mathrm{ET}$ McGill*, ${ }^{2} \mathrm{D}$ Marks, ${ }^{3} \mathrm{M}$ Petticrew, ${ }^{3} \mathrm{M}$ Egan. ${ }^{1}$ Deaprtment of Health Services, Research and Policy, London School of Hygiene and Tropical Medicine, London, UK; ${ }^{2}$ Department of Public Health, Environments and Society, London School of Hygiene and Tropical Medicine, London, UK

\subsection{6/jech-2020-SSMabstracts. 181}

Background English local authorities (LAs) have certain powers they can utilise to help them tackle alcohol-related harms. One such power is the Late Night Levy (LNL) which uses a 'polluter pays' principle whereby the LA charges alcohol retailers a fee for selling alcohol after midnight. Public health evaluators are increasingly considering how concepts from complexity science and systems thinking might shed light on how interventions can affect health in complex, real world settings such as local alcohol systems. This study aims to explore how the LNL acts as an event within the alcohol system, with a particular emphasis on understanding how the intervention, actors and the system as a whole adapt and coevolve in response.

Methods We conducted a process evaluation, using a complex systems perspective, in one LA. We included a range of system actors and utilised qualitative data generation methods. We interviewed LNL implementers and alcohol consumers $(n=21)$, conducted observations in pubs and during community-safety patrols (34.5 hours) and reviewed documents on the LNL and the broader system $(n=15)$. We conducted a deductive thematic analysis, applying concepts from systems thinking and complexity science to: 1) describe the system into which the LNL is introduced; and 2) identify emergent system outcomes.

Results The LNL operates within a complex system consisting of diverse actors with competing goals; these include the local political system and statutory functions, the retail sector, the third sector, residents and users of the nighttime economy. Early analyses identified a number of system responses stemming from the intervention, which include: 1) retailer adaptation to the levy; 2) new and evolving relationships between retailers, the police, community-safety officers and LA officers; 3) changes in venue management; and 4) the evolution of the intervention itself in response to broader system feedback. These adaptations are hypothesised to affect alcohol-related health and social harms. However, the intervention also interacts with wider system influences on public health, for example local government budgetary constraints during a period of central government cuts.

Discussion Conceptualising the LNL as an event within a complex system allows us to analyse its trajectory as it embeds within, and influences the broader system in which it is located. Further fieldwork and analyses are ongoing and focus on sampling additional system actors and exploring the influence of the vertical systems (e.g. national policy) which shape local responses to alcohol-related harms.

This study is funded by the National Institute for Health Research (NIHR) School for Public Health Research (Grant Reference Number PD-SPH-2015). The views expressed are those of the author(s) and not necessarily those of the NIHR or the Department of Health and Social Care.

\section{P90 HEALTH-JUSTICE PARTNERSHIPS: A SYSTEMATIC SCOPING REVIEW OF INTERNATIONAL EVIDENCE ON IMPACT}

${ }^{1} \mathrm{~S}$ Beardon*, ${ }^{2} \mathrm{C}$ Woodhead, ${ }^{1} \mathrm{~S}$ Cooper, ${ }^{3} \mathrm{H}$ Genn, ${ }^{1} \mathrm{R}$ Raine. ${ }^{1}$ Department for Applied Health Research, University College London, London, UK; ${ }^{2}$ Department of Psychological Medicine, King's College London, London, UK; ${ }^{3}$ Faculty of Laws, University College London, London, UK

\subsection{6/jech-2020-SSMabstracts. 182}

Background Social welfare legal problems impact negatively on mental and physical health. These include issues with welfare benefits, debt, homelessness, family and employment. Services providing legal assistance have developed collaborations with healthcare across the UK and the globe. These 'health-justice partnerships' aim to support health of the most disadvantaged groups, tackling interconnected challenges of health inequalities and access to justice. International evidence on the impacts of these partnerships has never previously been reviewed. This study interrogates the state of current evidence and provides new insights from international practice.

Methods A systematic scoping review of international literature was undertaken. Both academic and grey literature was included from OECD countries, 1995-2018. The review 